\title{
Erratum to: Clinical competence in the surgery of rectal cancer: the Italian Consensus Conference
}

\author{
Micaela Piccoli • Ferdinando Agresta • Vincenzo Trapani • Casimiro Nigro • Vito Pende - Fabio Cesare Campanile • \\ Nereo Vettoretto • Enrico Belluco • Paolo Pietro Bianchi • Davide Cavaliere • Giuseppe Ferulano • Filippo La Torre • \\ Marco Maria Lirici • Roberto Rea • Gianni Ricco • Elena Orsenigo • Simona Barlera • Emanuele Lettieri • \\ Giovanni Maria Romano • on behalf of The Italian Surgical Societies Working Group \\ Published online: 17 June 2014 \\ (C) Springer-Verlag Berlin Heidelberg 2014

\section{Erratum to: Int J Colorectal Dis} \\ DOI 10.1007/s00384-014-1887-x
}

The original version of this article unfortunately contains incorrect presentation of author names. The First and Family Name of the authors were mistakenly interchanged. The correct presentation of author names is presented above.

The online version of the original article can be found at http://dx.doi.org/ 10.1007/s00384-014-1887-x.

\section{Piccoli $\cdot$ V. Trapani}

Department of General and Emergency Surgery, NOCSAE, Modena, Italy

\section{F. Agresta $(\bowtie)$}

UOC di Chirurgia Generale, Presidio Ospedaliero di Adria (RO),

ULSS 19 del Veneto, Adria, RO, Italy

e-mail: fagresta@libero.it

\section{Nigro}

General Surgery Unit, University of Rome Tor Vergata, Viale

Oxford, 81, 00133 Rome, Italy

\section{Pende}

Department of Surgery Minimally Invasive Laparoscopic and Robotic

Surgery Unit, San Giovanni Addolorata Hospital, Rome, Italy

\section{F. C. Campanile}

Surgical Division, San Giovanni Decollato Andosilla Hospital (VT),

Civita Castellana, Italy

\section{N. Vettoretto}

Laparoscopic Surgical Unit, M.Mellini Hospital, Chiari, BS, Italy

E. Belluco

Surgery Unit, Monselice Hospital, PD ULSS, Veneto, Italy

\section{P. P. Bianchi}

Minimally-Invasive Surgery Unit, European Institute of Oncology, via Ripamonti 435, 20141 Milan, Italy

\section{Cavaliere}

Unit of Oncological Surgery and Advanced Therapies,

“Morgagni-Pierantoni”' Hospital—AUSL Forlì, Forlì, Italy

\section{G. Ferulano}

General and Minimally Invasive Surgery, University Department of Public Health University of Naples "Federico II", Naples, Italy

\section{F. La Torre}

Emergency Surgical Department at Policlinico "Umberto I", "La

Sapienza" University of Rome, Rome, Italy

M. M. Lirici

"San Giovanni" Hospital, Rome, Italy

R. Rea

U.O. Chirurgia Generale e Oncologica, Clinica Mediterranea,

Naples, Italy

G. Ricco

U.O. Malattie dell'Apparatro Digerente, Casa di Cura Villa del Sole, Salerno, Italy

E. Orsenigo

Department of Surgery at the Scientific Institute "San Raffaele",

"Vita-Salute San Raffaele" University, Milan, Italy

S. Barlera

Laboratory of Medical Statistics, Department of Cardiovascular Research, IRCCS MARIO NEGRI, Milan, Italy

E. Lettieri

Health Technology Assessment, Politecnico of Barlera, Milan, Italy

G. M. Romano

Department G.I. Oncology, National Cancer Institute and "G.

Pascale", Naples, Italy 